\title{
Dimensionamento do número de amostras para avaliação da fertilidade do solo
}

\section{The numbers of sample design for evaluation of the soil fertility}

\author{
Danilo Eduardo Rozane ${ }^{1 *}$; Liliane Maria Romualdo²; \\ José Frederico Centurion ${ }^{3}$; José Carlos Barbosa ${ }^{4}$
}

\begin{abstract}
Resumo
A determinação da quantidade de unidades amostrais que irão compor a amostra exprimirá a otimização da mão-de-obra, além de diminuir erros inerentes ao laudo de recomendação e avaliação da fertilidade do solo. Com este trabalho se objetivou determinar em três sistemas de uso e manejo do solo, o dimensionamento do número de unidades amostrais, necessárias a formar a amostra composta, para avaliação da fertilidade do solo. Concluiu-se que o número de unidades amostrais necessário a compor a amostra composta para determinação dos atributos de matéria orgânica, $\mathrm{pH}, \mathrm{P}, \mathrm{K}, \mathrm{Ca}, \mathrm{Mg}, \mathrm{H}+\mathrm{Al}$ e saturação por bases do solo variam conforme o uso e manejo do solo e o erro aceitável para a estimativa da média. Para uma mesma profundidade de coleta, o aumento do número de unidades amostrais, reduziu o erro porcentual na estimativa da média, permitindo a recomendação de 14, 14 e 11 unidades amostrais no manejo com mata nativa, pastagem e milho, respectivamente, para um erro de $20 \%$ na estimativa da média.
\end{abstract}

Palavras-chave: Amostragem de solo, unidades amostrais, milho, Panicum, mata, variabilidade horizontal

\begin{abstract}
The determination of the amount of sample units that will compose the sample express the optimization of the workforce, and reduce errors inherent in the report of recommendation and evaluation of soil fertility. This study aimed to determine in three systems use and soil management, the numbers of units samples design, needed to form the composed sample, for evaluation of soil fertility. It was concluded that the number of sample units needed to compose the composed sample to determination the attributes of organic matter, $\mathrm{pH}, \mathrm{P}, \mathrm{K}, \mathrm{Ca}, \mathrm{Mg}, \mathrm{Al}$ and $\mathrm{H}+\mathrm{Al}$ and base saturation of soil vary by use and soil management and error acceptable to the mean estimate. For the same depth of collected, increasing the number of sample units, reduced the percentage error in estimating the average, allowing the recommendation of 14,14 and 11 sample in management with native vegetation, pasture cultivation and corn, respectively, for a error $20 \%$ on the mean estimate.
\end{abstract}

Key words: Soil simpling, units samples, corn, Panicum, native vegetation, horizontal variability

1 Pós-Doutorando. Faculdade de Ciências Agrárias e Veterinárias de Jaboticabal, FCAV, Universidade Estadual Paulista "Júlio de Mesquita Filho", Unesp/FCAV, Departamento de Solos e Adubos, Via Acesso Prof. Paulo Donato Castellane, s/n, Jaboticabal SP, CEP: 14884-900. E-mail: danilorozane@yahoo.com.br

2 Zootecnista, Doutoranda, Universidade de São Paulo. Av. Duque de Caxias Norte, 225 Departamento de Zootecnia, Pirassununga - SP, CEP: 13635-900 -. E-mail: lilianeromualdo@yahoo.com.br

3 Prof., Dr., Faculdade de Ciências Agrárias e Veterinárias de Jaboticabal, FCAV, UNESP/FCAV, Departamento de Solos e Adubos. E-mail: jfcentur@fcav.unesp.br

${ }^{4}$ Prof., Titular, Faculdade de Ciências Agrárias e Veterinárias de Jaboticabal, FCAV, UNESP/FCAV, Departamento de Ciências Exatas. E-mail: jcbarbosa@fcav.unesp.br

* Autor para correspondência

Recebido para publicação 23/12/09 Aprovado em 28/01/11 


\section{Introdução}

No manejo da fertilidade do solo a amostragem é, sem dúvida, a mais sensível e sujeita a erros. $\mathrm{Na}$ amostragem, a amostra que comumente é utilizada para representar a área a ser corrigida e, ou, adubada, é a "amostra composta ou amostra". Cada amostra é formada pela mistura homogênea de um número predefinido de "unidades amostrais ou amostras simples" de pequeno volume (GUARÇONI et al., 2006).

Pode-se afirmar que a amostra mais adequada é aquela que representa o melhor possível a área a ser avaliada, com um mínimo de unidades amostrais realizadas para atender a este objetivo; assim, a análise estatística auxilia na indicação de um número mínimo de pontos suficientes para reduzir a variação dos resultados a um nível aceitável. Com isso, tem-se uma amostra representativa da população para que os resultados da análise de terra tenham validação técnica e científica, e que recursos não sejam empregados desnecessariamente e, ou, evitando amostragem não representativa.

Além dos aspectos inerentes ao solo (Pedologia, fertilidade, ...), outro ponto que tem suscitado dúvidas diz respeito ao número de unidades amostrais que venham a compor a amostra e assim, garantir a representatividade da área homogênea. Uma gleba considerada tradicionalmente como homogênea é definida como uma porção de solo que expressa um arranjo homogêneo dos fatores limitantes da produção pelos quais uma determinada taxa de um insumo específico é apropriada (DOERGE, 2000).

Entretanto não há consonância quanto ao número amostras necessárias para se estimar a fertilidade média (CHITOLINA, 1982), com variações significativas. A exemplo de Leo (1983), citado por Peck e Melsted (1973), que calculou ser necessário de 2 a 500 unidades amostrais para se ter uma amostra representativa de um talhão homogêneo. Boletins de extensão para condições brasileiras recomendam a coleta de 20 unidades amostrais para compor uma amostra (RAIJ et al., 1997). Em trabalhos mais recentes considerando-se o atributo mais limitante e o erro porcentual na estimativa da média de $20 \%$ o número de unidades amostrais (amostras simples) pode variar de 50 a 188 (SILVEIRA et al., 2000); de 11 a 183 (SCHLINDWEIN; ANGHINONI, 2002); de 20 a 39 (GUARÇONI et al., 2006) e de 5 a 20 (GUARÇONI et al., 2007).

A análise de terra é uma ferramenta consagrada, porém, há necessidade de se empregá-la adequadamente, deste modo, conhecer os fatores que a limitam permite a adoção de programas de calagem e adubação mais adequados, com resultados favoráveis ao aumento da produtividade e, consequentemente, do lucro do produtor.

Buscando maiores esclarecimentos sobre o assunto, objetivou-se, neste estudo determinar, em três sistemas de uso e manejo do solo o dimensionamento do número de unidades amostrais, necessárias para formar uma amostra composta, para fins de avaliação da fertilidade do solo.

\section{Material e Métodos}

O estudo foi realizado, em um Latossolo Vermelho Distrófico, típico, textura média, A-Moderado, hipoférrico e muito profundo (Haplustox) com relevo suave ondulado (EMBRAPA, 1999). As coordenadas geográficas da área experimental são $21^{\circ} 14^{\prime} 07^{\prime}$ ' de latitude sul e $48^{\circ} 16^{\prime} 35^{\prime \prime}$ de longitude oeste, e altitude de 595m, localizada no Pólo Regional de Desenvolvimento Tecnológico dos Agronegócios de Alta Mogiana no município de Colina, SP. A precipitação média anual é de 1262 $\mathrm{mm}$ e a temperatura média, de $24,8^{\circ} \mathrm{C}$. O clima é tropical chuvoso (Aw), pelo critério de classificação climática de Köppen.

Os sistemas de uso e manejo utilizados no dimensionamento do número de amostras para avaliação da fertilidade do solo constituem-se por: Mata nativa, pastagem e milho para grão, sendo que em cada manejo a gleba utilizada na amostragem foi de 10 hectares. Foram coletadas 30 unidades 
amostrais tomadas ao acaso, com auxílio de um trado tipo holandês, em cada área e situação de manejo, na profundidade de $0-0,10 \mathrm{~m}$.

A camada de amostragem de $0-0,10 \mathrm{~m}$ de profundidade foi estabelecida pela recomendação de Cantarutti et al. (1999) para pastagens implantadas, estendida para as demais áreas, visto que a coleta de solo em profundidades distintas provoca a mistura de camadas com diferentes teores, especialmente quando os gradientes de concentração são muito elevados nas camadas superficiais, em especial de culturas sob o sistema de semeadura direta (ANGHINONI; SALET, 1998), procedimento este que pode-se estender ao solo de mata nativa. Além disso, Silveira e Stone (2002) comprovaram que a mistura de camadas de solo com diferentes teores de nutrientes, além de diluir o valor médio dos atributos avaliados, gera maiores estimativas de variabilidade (C.V.) para um mesmo valor de t e um mesmo erro na estimativa da média $(d)$, justificando desta maneira a homogeneidade na coleta da profundidade das amostras de solo.

A área de pastagem (Panicum maximum cv. Capim Tanzânia) foi implantada em 1997 sendo manejada desde então com bovinos de corte. A gleba cultivada com milho sob irrigação (Pivô central), que anteriormente era cultivado com Capim Tanzânia, iniciou-se em 2006. Para sua implantação, realizouse o preparo do solo, com duas gradagens pesadas (16 discos de 32 polegadas), mais uma gradagem de nivelamento ( 24 discos de 28 polegadas), sendo este manejo também realizado na implantação do Capim Tanzânia em 1997. Especificamente para o milho, a calagem foi realizada em abril de 2006 com aplicação de $6 \mathrm{t}$ ha ${ }^{-1}$ de calcário dolomítico, além de receber na adubação de plantio $1000 \mathrm{~kg} \mathrm{ha}^{-1}$ da formulação 20-05-20. Já na adubação de cobertura, realizada em 15 de junho de 2006, utilizou-se 1100 $\mathrm{kg} \mathrm{ha}^{-1}$ da fórmula 14-07-28, incorporada através do cultivador DMB. O milho foi colhido em maio de 2007, alcançando 3,5 toneladas por hectare.

$\mathrm{Na}$ mata nativa, as unidades amostrais foram dispostas ao acaso, com caminhamento em zigue- zague de forma homogênea, procedimento este seguido também na pastagem, que apresentava dez anos desde a instalação. Após a instalação da cultura do milho, a mesma vem sendo conduzida em sistema de semeadura direta, sendo as unidades amostrais de solo coletadas logo após a colheita, na entrelinha de semeadura, o qual era cultivado no espaçamento de $0,90 \mathrm{~m}$ entre-linhas. As áreas utilizadas no estudo localizam-se a aproximadamente, $50 \mathrm{~m}$ de distância entre si.

As determinações analíticas ( $\mathrm{pH} \mathrm{em} \mathrm{CaCl}_{2}, \mathrm{P}_{\text {resina' }}$, M.O., K, Ca, Mg e H+Al) e os parâmetros calculados ( $\mathrm{SB}, \mathrm{CTC}$ e V\%) nos diferentes sistemas de uso e manejo do solo para fins de fertilidade seguiram a os métodos descritos por Raij et al. (2001). Para cada atributo foi calculado a média, o desvio-padrão, o erro padrão da média e o coeficiente de variação. $\mathrm{O}$ número de unidades amostrais para estimar os parâmetros de uma população infinita para um nível de precisão desejado foi baseado no intervalo de confiança para a média, dado pela seguinte equação, descrita em Thompson (1992):

$$
n=\frac{t^{2} s^{2}}{d^{2} m^{2}}
$$

Onde: $n$ é o tamanho da amostra; $t$ é o valor da distribuição $t$ de Student a $5 \%$ de probabilidade; $s^{2}$ é a variância amostral; $d$ é o erro na estimativa da média; $m$ é a média amostral.

\section{Resultados e Discussão}

De acordo com Pimentel-Gomes e Garcia (2002), a variabilidade de um atributo pode ser classificada de acordo com a magnitude do seu coeficiente de variação, que pode ser: (a) baixo, quando menor que $10 \%$; (b) média, quando entre 10 e $20 \%$; (c) alta, quando entre 20 e $30 \%$; e (d) muito alta, se maior que $30 \%$. Com isso, pode-se observar que no presente estudo o atributo $\mathrm{pH}$ apresentou coeficientes de variação baixos, em todas as áreas, variando de $4,3 \%$ a $8,1 \%$ concordando com os 
trabalhos de Prevedello (1987) e Silveira et al. (2000). As demais variáveis estudadas encontramse na classe (c) (Tabela 1); coeficientes de variação semelhantes dentre os mesmos atributos avaliados foram também observados por Silveira et al. (2000), Guarçoni et al. (2006, 2007). Entretanto, Barreto, Novais e Braga (1974) enfatizam que para determinar o número de unidades amostrais, afim de formar uma amostra representativa, a coleta do maior número de unidades amostrais aumenta a confiabilidade ou exatidão dessa média obtida, pela diminuição do valor de $d$.

Com exceção do fósforo nos diferentes sistemas de uso e manejo do solo, os demais valores constantes das análises de solo (Tabela 1) são considerados médios a alto nas classes de interpretação de acordo com classificação de Raij et al. (1997).

Em termos gerais, a variabilidade dos atributos avaliados, estimatida pelo número de unidades amostrais necessárias a compor uma amostra, seguiu a ordem: $\mathrm{pH}<\mathrm{V}<$ M.O. $<\mathrm{P}<\mathrm{H}+\mathrm{Al}<\mathrm{Mg}^{2+}<\mathrm{Ca}^{2+}$ $<\mathrm{K}$. Resultados semelhantes foram observados por diversos autores Barreto, Novais e Braga (1974); Anghinoni e Salet (1998); Schlindwein e Anghinoni (2000); Guarçoni et al. (2006, 2007), a exceção do $\mathrm{P}$ que apresentou uma menor variabilidade. A causa desta influência pode ser explicada uma vez que a amostragem no milho deu-se na entre linha da cultura, não sofrendo influencia da adubação localizada no sulco, além do sistema de semeadura direta ter sido recentemente implantado, estando, portanto, semelhantemente à pastagem e a mata nativa, que já se encontram sem aporte de fertilizantes. Associado a isto está a coleta realizada na camada de $0-0,1 \mathrm{~m}$ que, como já discutido, minimiza o efeito de variabilidade. Também, segundo Guarçoni et al. (2007), a explicação pode estar associada ao equipamento de coleta utilizado, que incorpora no volume de solo coletado as variações horizontais a curtas distâncias, ou seja, diminuindo, consequentemente, a estimativa da variabilidade.

A quantidade de unidades amostrais necessárias para formar uma amostra representativa do talhão homogêneo amostrado está condicionada ao nível de precisão desejado, definido em função dos objetivos da amostragem. Para a amostragem de solo sem, contudo, imprimir aos resultados um erro elevado em torno da verdadeira média populacional, é admissível que um erro de até $20 \%$ em torno da média, como preconizam Montesinos et al. (2002); Guarçoni et al. (2006); Oliveira et al. (2007); Amaro Filho et al. (2007) e Guarçoni et al. (2007).

Para as três situações de uso e manejo do solo a estimativa do número de unidades amostrais necessárias para compor a amostra diminuiu a medida que se aumentou o $d$. O dimensionamento da amostra esteve associada com o tipo de uso e manejo do solo e conforme o atributo de fertilidade (Tabela 1). Dentre os atributos de fertilidade avaliados, o que estimou o maior número de unidades amostrais necessárias a compor a amostra esta associado ao potássio, corroborando com Silveira et al. (2000), uma vez que este atributo apresentou a maior variabilidade dentre todas as estudadas, necessitando portanto do maior número de unidades amostrais para compor a amostra (Tabela 1). Dessa forma, nas condições em que o presente estudo foi desenvolvido, pode-se recomendar a coleta de 14,14 e $11(d=20 \%)$ a 55,55 e $45(d=10 \%)$ unidades amostrais para compor a amostra, para as situações de manejo: mata nativa, pasto e milho, respectivamente.

A literatura internacional mostra que não há concordância entre os autores quanto ao tamanho da área a amostrar e o número de amostras necessárias para se estimar a fertilidade média (CHITOLINA, 1982), com variações bastante significativas. Por exemplo, para Barker e Steyn (1956), citados por Chitolina (1982), em áreas tão pequenas quanto 100 $\mathrm{m}^{2}$, são necessárias 100 unidades amostrais para representação satisfatória dos teores de N, P e K do solo. Já Leo (1983), citado por Peck e Melsted (1973), calculou que são necessárias desde 2 até 500 unidades amostrais para se ter uma amostra representativa, sendo que o número varia de acordo com o nutriente. 
Para condições brasileiras, Raij et al. (1997) recomenda a coleta de 20 unidades amostrais para compor uma amostra. Em trabalhos mais recentes, considerando-se o atributo mais limitante e o $d$ de até $20 \%$, o número de unidades amostrais pode variar de 50 a 188 (SILVEIRA et al., 2000); de 11 a 183 (SCHLINDWEIN; ANGHINONI, 2002); de 20 a 39 (GUARÇONI et al., 2006) e de 5 a 20 (GUARÇONI et al., 2007), evidenciando também a peculiaridade de cada condição experimental.

Dentre as situações de manejo, a maior amplitude observada foi na mata nativa seguida da pastagem, o que explica o maior coeficiente de variação e os maiores valores no desvio padrão nestes tratamentos em relação à área do milho (Tabela 1 ). Em razão do não-revolvimento do solo, espera-se maior variabilidade dos dados neste tratamento, como também observou Silveira et al. (2000) em sistema de semeadura direta há cinco anos. Segundo Souza (1992), a prática de revolver o solo possibilita menor variabilidade, horizontal e vertical, nos teores de matéria orgânica e nutrientes na camada arada do solo do que o plantio direto, causando desta maneira a diluição e homogeneização do nutriente com maior volume de solo. Silveira et al. (2000) enfatiza que em sistemas de semeadura direta há concentração dos teores do elemento na primeira camada, aumentando assim a estimativa do número de unidades amostrais necessárias a compor a amostra, o que aproxima dos tratamentos pastagem e mata nativa.

Tabela 1. Estatística descritiva e estimativa do número de unidades amostrais necessárias a compor a amostra para avaliação dos atributos químicos do solo, em função do erro na estimativa da média, em três situações de uso e manejo do solo.

\begin{tabular}{|c|c|c|c|c|c|c|c|c|c|c|c|c|}
\hline \multirow{4}{*}{$\begin{array}{l}\% \text { Erro na } \\
\text { estimativa } \\
\text { da média } d \\
1\end{array}$} & \multicolumn{12}{|c|}{ Situações de manejo } \\
\hline & Mata & Pasto & Milho & Mata & Pasto & Milho & Mata & Pasto & Milho & Mata & Pasto & Milho \\
\hline & \multicolumn{3}{|c|}{---- pH $\left(\mathrm{CaCl}_{2}\right)$---- } & \multicolumn{3}{|c|}{---- $\mathrm{MO}\left(\mathrm{g} \mathrm{dm}^{-3}\right)$---- } & \multicolumn{3}{|c|}{$--\mathrm{P}_{\text {resina }}\left(\mathrm{mg} \mathrm{dm}^{-3}\right)--$} & \multicolumn{3}{|c|}{$--\mathrm{K}\left(\mathrm{mmol}_{\mathrm{c}} \mathrm{dm}^{-3}\right)--$} \\
\hline & 432 & 123 & 291 & 2866 & 1324 & 1965 & $2907^{\mathrm{r}}$ & 2296 & 2796 & 5463 & $5498^{c}$ & 4541 \\
\hline 5 & 17 & 5 & 12 & 115 & 53 & 79 & 116 & 92 & 112 & 219 & 220 & 182 \\
\hline 10 & 4 & 1 & 3 & 29 & 13 & 20 & 29 & 23 & 28 & 55 & 55 & 45 \\
\hline 15 & 2 & 1 & 1 & 13 & 6 & 9 & 13 & 10 & 12 & 24 & 24 & 20 \\
\hline 20 & 1 & 1 & 1 & 7 & 3 & 5 & 7 & 6 & 7 & 14 & 14 & 11 \\
\hline 25 & 1 & 1 & 1 & 5 & 2 & 3 & 5 & 4 & 4 & 9 & 9 & 7 \\
\hline 30 & 1 & 1 & 1 & 3 & 1 & 2 & 3 & 3 & 3 & 6 & 6 & 5 \\
\hline$\hat{m}$ & 5,3 & 5,1 & 5,2 & 20 & 20 & 16 & 8 & 5 & 7 & 3,4 & 2,2 & 2,3 \\
\hline$s$ & 0,43 & 0,22 & 0,35 & 4,08 & 2,77 & 2,82 & 1,78 & 0,94 & 1,52 & 0,98 & 0,63 & 0,59 \\
\hline$s(\hat{m})$ & 0,19 & 0,10 & 0,15 & 1,83 & 1,24 & 1,26 & 0,79 & 0,42 & 0,68 & 0,44 & 0,28 & 0,27 \\
\hline \multirow{2}{*}{$C V$} & 8,1 & 4,3 & 6,6 & 20,8 & 14,2 & 17,2 & 21,0 & 18,6 & 20,6 & 28,8 & 28,8 & 26,2 \\
\hline & \multicolumn{3}{|c|}{-- $\mathrm{Ca}\left(\mathrm{mmol}_{\mathrm{c}} \mathrm{dm}^{-3}\right)--$} & \multicolumn{3}{|c|}{-- $\mathrm{Mg}\left(\mathrm{mmol} \mathrm{dm}^{-3}\right)--$} & \multicolumn{3}{|c|}{$\mathrm{H}+\mathrm{Al}\left(\mathrm{mmol}_{\mathrm{c}} \mathrm{dm}^{-3}\right)$} & \multicolumn{3}{|c|}{--------- V \% --------- } \\
\hline 1 & 5175 & $1886^{c}$ & 5044 & 4813 & $1360^{c}$ & 4660 & 4176 & 882 & 2899 & 1784 & 824 & 2049 \\
\hline 5 & 207 & 75 & 202 & 193 & 54 & 186 & 167 & 35 & 116 & 71 & 33 & 82 \\
\hline 10 & 52 & 19 & 50 & 48 & 14 & 47 & 42 & 9 & 29 & 18 & 8 & 20 \\
\hline 15 & 23 & 8 & 22 & 21 & 6 & 21 & 19 & 4 & 13 & 8 & 4 & 9 \\
\hline 20 & 13 & 5 & 13 & 12 & 3 & 12 & 10 & 2 & 7 & 4 & 2 & 5 \\
\hline 25 & 8 & 3 & 8 & 8 & 2 & 7 & 7 & 1 & 5 & 3 & 1 & 3 \\
\hline 30 & 6 & 2 & 6 & 5 & 2 & 5 & 5 & 1 & 3 & 2 & 1 & 2 \\
\hline$\hat{m}$ & 21 & 12 & 17 & 16 & 9 & 11 & 23 & 23 & 22 & 64 & 49 & 57 \\
\hline$s$ & 5,90 & 1,97 & 4,62 & 4,33 & 1,22 & 3,01 & 5,67 & 2,70 & 4,68 & 10,46 & 5,46 & 10,07 \\
\hline$s(\hat{m})$ & 2,64 & 0,88 & 2,07 & 1,94 & 0,55 & 1,35 & 2,54 & 1,21 & 2,09 & 4,68 & 2,44 & 4,50 \\
\hline$C V$ & 28,0 & 16,9 & 27,6 & 27,0 & 14,3 & 26,6 & 25,1 & 11,6 & 20,9 & 16,4 & 11,2 & 17,6 \\
\hline
\end{tabular}

$\hat{m}$ é a estimativa da média amostral; $S$ é o desvio-padrão; $s(\hat{m})$ é o erro-padrão da média; e $C V$ é o coeficiente de variação. 
$\mathrm{Na}$ mata nativa possivelmente há maiores variações horizontais a curtas distâncias, o que provavelmente possa ser explicado pela deposição pontual da fauna e flora. Entretanto, de maneira global para a planta, essa avaliação possa não ser intrínseca, pois, como evidencia Jackson (1976), a variabilidade a curtas distâncias no solo não dificulta o crescimento e o desenvolvimento das plantas, uma vez que a extensão do sistema radicular é suficiente para explorar áreas com diferentes teores de nutrientes.

Com base nas recomendações de adubação de Raij e Cantarella (1997) para milho grão, e de Werner et al. (1997) para adubação de manutenção de pastagem, e tendo-se como referência a determinação do $\mathrm{K}$, que estimou o maior número de unidades amostrais necessárias a compor a amostra, na maior tolerância aceitável $(d=20 \%)$, seria necessário realizar 14 e 13 unidades amostrais, respectivamente para a pastagem e para o milho (Tabela 1).

No entanto, observa-se pelos intervalos de interpretação das classes de fertilidade disponíveis para recomendação de adubação que não seriam necessários mais de 1 e 7 unidades amostrais para compor a amostra para pastagem e milho, respectivamente, com o objetivo de realizar a recomendação de calagem, adubação fosfatada e potássica, as quais são dependentes da análise de solo. Resultados semelhantes em duas áreas com a mesma dimensão foram apresentados por Chitolina (1982), onde o autor concluiu que são necessárias 10 unidades amostrais para a Terra Roxa Estruturada (atual Nitossolo) e o Latossolo Vermelho Escuro - fase arenosa (atual Latossolo Vermelho), a uma confiabilidade de $80 \%$ e com uma variação em torno da média de $20 \%$, para se fazer a recomendação de adubação para milho de acordo com o Boletim 209 do Instituto Agronômico de Campinas (1977), referencial da época.

\section{Conclusões}

O número de unidades amostrais necessário a compor a amostra para os atributos de matéria orgânica, $\mathrm{pH}, \mathrm{P}, \mathrm{K}, \mathrm{Ca}, \mathrm{Mg}, \mathrm{H}+\mathrm{Al}$ e saturação por bases do solo variam conforme o uso e manejo do solo e o erro aceitável para a estimativa da média.

Para uma mesma profundidade de coleta, o aumento do número de unidades amostrais reduziu o erro porcentual na estimativa da média, permitindo a recomendação de 14, 14 e 11 unidades amostrais no manejo com mata nativa, pastagem e milho, respectivamente, para um erro de $20 \%$ na estimativa da média.

\section{Referências}

AMARO FILHO, J.; NEGREIROS, R. F. D. de; ASSIS JÚNIOR, R. N. D. E.; MOTA, J. C. A. Amostragem e variabilidade espacial de atributos físicos de um latossolo vermelho em Mossoró, RN. Revista Brasileira de Ciência do Solo, Viçosa, MG, v. 31, n. 3, p. 415-422, 2007.

ANGHINONI, I.; SALET, R. L. Amostragem do solo $e$ as recomendações de adução e calagem no sistema plantio direto. In: NUERNBERG, N. J. (Ed.). Conceitos e fundamentos do sistema plantio direto. Lages: SBCSNRS. p. 27-52, 1998.

BARRETO, A. C.; NOVAIS, R. F.; BRAGA, J. M. Determinação estatística do número de amostras simples de solo por área para avaliação de sua fertilidade. Ceres, Viçosa, MG, v. 21, n. 114, p. 142-147, 1974.

CANTARUTTI, R. B.; MARTINS, C. E.; CARVALHO, M. M.; FONSECA, D. M. da; ARRUDAS, M. L.; VILELAS, H.; OLIVEIRA, F. T. T. Pastagens. In: RIBEIRO, A. C.; GUIMARÃES, P. T. G.; ALVAREZ, V. V. H. (Ed.). Recomendações para uso de corretivos e fertilizantes em Minas Gerais - $5^{a}$ aproximação. Viçosa, MG: CFSEMG/UFV. p. 332-341, 1999.

CHITOLINA, J. C. Contribuição de alguns fatores nos resultados da análise química de terra e seus efeitos nas recomendações de adubação e calagem. 1982. Tese (Doutorado em Solos e Nutrição de Plantas) - Escola Superior de Agricultura "Luiz de Queiroz”, Piracicaba.

DOERGE, T. A. Management zones concepts. Sitespecific management guidelines. Atlanta, USA: Potash and Phosphate Institute, 2000. v. 2, p. 1-4. 
EMPRESA BRASILEIRA DE PESQUISA AGROPECUÁRIA - EMBRAPA. Centro Nacional de Pesquisa de Solos (Rio de Janeiro, RJ). Sistema Brasileiro de Classificação de Solos. Rio de Janeiro: EMBRAPASolos, 1999. 412 p. (EMBRAPA-Solos. Documentos, $15)$.

GUARÇONI, M. A.; ALVAREZ, V. V. H.; NOVAIS, R. F.; CANTARUTTI, R. B.; LEITE, H. G.; FREIRE, F. M. Diâmetro de trado necessário à coleta de amostras num Cambissolo sob plantio direto ou sob plantio convencional antes ou depois da aração. Revista Brasileira de Ciência do Solo, Viçosa, MG, v. 31, n. 5, p. 947-959, 2007.

GUARÇONI, M.A.; ALVAREZ, V. V. H.; NOVAIS, R. F.; CANTARUTTI, R. B.; LEITE, H. G.; FREIRE, F. M. Definição da dimensão do indivíduo solo e determinação do número de amostras simples necessário à sua representação. Revista Brasileira de Ciência do Solo, Viçosa, MG, v. 30, n. 6, p. 943-954, 2006.

INSTITUTO AGRONÔMICO DE CAMPINAS - IAC. Tabelas de adubação e calagem das principais culturas econômicas do Estado de São Paulo. Campinas: Instituto Agronômico, 1977. 198 p. (Boletim, n. 209).

JACKSON, M. L. Analisis quimico de suelos. Barcelona: Omega, 1976. 662 p.

MONTESINOS, M. G.; CÁMARA, J. M. F.; TOMÉ, J. M.; CÁMARA, M. A. O.; GARCÍA, F. H.; VICENTE, R. M.; DÍEZ, M. B. Número de sub muestras necesarias para la obtención de una muestra de suelo representativa. Agrícola Vergel, Valencia, v. 241, n. 1, p. 9-18, 2002.

OLIVEIRA, F. H. T. de; ARRUDA, J. A. de; SILVA, I. de F. da; ALVES, J. do C. Amostragem para avaliação da fertilidade do solo em função do instrumento de coleta das amostras e de tipos de preparo do solo. Revista Brasileira de Ciência do Solo, Viçosa, MG, v. 31, n. 5, p. 973-983, 2007.

PECK, R.; MELSTED, S. W. Field sampling for soil testing. In: WALSH, L. M.; BEATON, J. D. (Ed.). Soil testing and plant analysis. Soil Science Society of America Journal, Madison, v. 37, n. 1, p. 67-73, 1973.

PIMENTEL-GOMES, F.; GARCIA, C. H. Estatística aplicada a experimentos agronômicos e florestais: exposição com exemplos e orientações para uso de aplicativos. Piracicaba: FEALQ, 2002. 309 p.

PREVEDELLO, B. M. S. Variabilidade espacial de parâmetros de solo e planta. 1987. (Tese de Doutorado em Solos e Nutrição de Plantas) - Escola Superior de Agricultura Luiz de Queiroz, Piracicaba.
RAIJ, B. van; ANDRADE, J. C.; CANTARELLA, H.; QUAGGIO, J. A. Análise química para avaliação da fertilidade de solo tropicais. Campinas: Instituto Agronômico, 2001. 285 p.

RAIJ, B. van; CANTARELLA, H. Milho para grãos e silagem. In: RAIJ, B. van; CANTARELLA, H.; QUAGGIO, J. A.; FURLANI, A. N. C. (Ed.). Recomendações de adubação e calagem para o Estado de São Paulo. Campinas: Instituto Agronômico/Fundação IAC, 1997, 285 p. (Boletim Técnico, 100).

RAIJ, B. van; CANTARELLA, H.; QUAGGIO, J. A.; FURLANI, A. N. C. (Ed.). Recomendações de adubação e calagem para o Estado de São Paulo. Campinas: Instituto Agronômico/Fundação IAC, 1997, 285 p. (Boletim Técnico, 100).

SCHLINDWEIN, J. A.; ANGHINONI, I. Tamanho da subamostra e representatividade da fertilidade do solo no sistema plantio direto. Ciência Rural, Santa Maria, RS, v. 32, n. 6, p. 963-968, 2002.

Variabilidade vertical de fósforo e potássio disponíveis e profundidade de amostragem do solo no sistema plantio direto. Ciência Rural, Santa Maria, RS, v. 30, n. 4, p. 611-617, 2000.

SILVEIRA, P. M. da; ZIMMERMANN, F. J. P.; SILVA, S. C. da; CUNHA, A. A. Amostragem e variabilidade espacial de características químicas de um latossolo submetido a diferentes sistemas de preparo. Pesquisa Agropecuária Brasileira, Brasília, DF, v. 35, n. 10, p. 2057-2064, 2000.

SILVEIRA, P. M.; STONE, L. F. Profundidade de amostragem do solo sob plantio direto para avaliação de características químicas. Revista Brasileira de Ciência do Solo, Viçosa, MG, v. 26, n. 1, p. 157-162, 2002.

SOUZA, L. S. Variabilidade espacial do solo em sistemas de manejo. 1992. Tese (Doutorado em Ciência do Solo) - Universidade Federal do Rio Grande do Sul, Porto Alegre.

THOMPSON, S. K. Sampling. New York: John Wiley, 1992. $343 \mathrm{p}$.

WERNER, J. C.; PAULINO, V. T.; CANTARELLA, H.; QUAGGIO, J. A.; ANDRADE, N. de O. Forrageiras. In: RAIJ, B. van, CANTARELLA, H.; QUAGGIO, J. A.; FURLANI, A. N. C. (Ed.). Recomendações de adubação e calagem para o Estado de São Paulo. Campinas: Instituto Agronômico, 1997. 285 p. (Boletim Técnico, 100). 
